International Mathematical Forum, Vol. 9, 2014, no. 11, 499 - 514

HIKARI Ltd, www.m-hikari.com

http://dx.doi.org/10.12988/imf.2014.4121

\title{
On the Existence of Certain Steiner Systems
}

\author{
Shuaa Aldhafeeri \\ Department of Mathematics \\ College of Basic Education \\ PAAET \\ Kuwait
}

Copyright (c) 2014 Shuaa Aldhafeeri. This is an open access article distributed under the Creative Commons Attribution License, which permits unrestricted use, distribution, and reproduction in any medium, provided the original work is properly cited.

\begin{abstract}
In this paper we prove the existence of certain Steiner systems. The results of this paper are divided into three sections: In section 1 , we investigate Steiner systems of type $S(3,4,8)$. In section 2 , we study Steiner systems of type $S(5,6,12)$, and in the last section we study Steiner systems of type $(5,8,24)$, the MOG and the Hexacode.
\end{abstract}

Mathematics Subject Classification: 20C15, 20E28

Keywords: Steiner system, Kitten, MOG

\section{Introduction}

Steiner systems were named after the $19^{\text {th }}$ century Swiss mathematician Jakob Steiner [10].

A Steiner system $S=S(\Omega, \mathcal{B})$ is a finite set $\Omega$; whose elements we call points, along with a set $\mathcal{B}$ of subsets of $\Omega$ called blocks, which for some integers $k$ and $t$, satisfy the following conditions:

1. Each block in $\mathcal{B}$ has size $k$;

2. Any set $X \subset \Omega$ of size $t \leqslant k$ is contained in exactly one block from $\mathcal{B}$.

If we put $v=|\Omega|$, then such a system is called an $S(t, k, v)$ Steiner system.

An automorphism of a Steiner system $S=S(t, k, v)$ is a permutation of $\Omega$ which permutes the blocks among themselves. We can count the number of $t$-subsets in two ways; 
- The number of $t$-subsets $=$ the number of $k$-blocks in $S \times$ the number of $t$-subsets contained in each $k$-block $=|S| \times\left(\begin{array}{l}k \\ t\end{array}\right)$.

- Also, the number of $t$-subsets $=\left(\begin{array}{l}v \\ t\end{array}\right)$.

Hence the number of blocks of $S(t, k, v)=\frac{\left(\begin{array}{l}v \\ t\end{array}\right)}{\left(\begin{array}{c}k \\ t\end{array}\right)}$.

Whenever we have a Steiner system $S(t, k, v)$, then taking the derived design (taking all blocks that contain a fixed element, and then discarding that element), we obtain an $S(t-1, k-1, v-1)$ from an $S(t, k, v)$.

Definition 1.1 Let $S=S(\Omega, \mathcal{B})$ be an $S(t, k, v)$ Steiner system. For any point $\omega$ of $S$ we can form a new Steiner system $S_{\omega}=\left(\Omega^{\prime}, \mathcal{B}^{\prime}\right)$ where $\Omega^{\prime}=\Omega \backslash \omega$ and $\mathcal{B}^{\prime}=\{B \backslash \omega \mid B \in \mathcal{B}$ and $\omega \in B\}$. Then the Steiner system $S_{\omega}$ is called the contraction of $S$ at $\omega$ and is an $S(t-1, k-1, v-1)$ Steiner system. Also, $S$ is then described as an extension of $S_{\omega}$.

Note that the Steiner systems $S(5,8,24), S(4,7,23), S(3,6,22), S(2,5,21)$, $S(5,6,12), S(4,5,11), S(3,4,10)$, and $S(2,3,9)$ are all unique [1].

However, it is far from clear that an $S(t, k, v)$ can be extended to as $S(t+$ $1, k+1, v+1)$. Indeed it may happen that an $S(t, k, v)$ exists but

$$
\left(\begin{array}{c}
k+1 \\
t+1
\end{array}\right) \chi\left(\begin{array}{l}
v+1 \\
t+1
\end{array}\right)
$$

Indeed no Steiner systems are known with $t$ larger than 5 [6].

\section{Results and Discussion}

The results of this paper are divided into three sections:

\subsection{Steiner system $S(3,4,8)$}

Proposition 2.1 A Steiner system $S(2,3,7)$ can be extended to a Steiner system $S(3,4,8)$.

Proof. Suppose that this Steiner system exists, then the number of tetrads in this Steiner system is $\frac{\left(\begin{array}{l}8 \\ 3\end{array}\right)}{\left(\begin{array}{l}4 \\ 3\end{array}\right)}=\frac{8193 \times 7 \times 6}{4 \times 3 \times 2}=14$. So, there are 14 tetrads of which $\frac{\left(\begin{array}{l}7 \\ 2\end{array}\right)}{\left(\begin{array}{l}3 \\ 2\end{array}\right)}=7$ of them contain a specific point and $14-7=7$ do not contain this point. For a given pair of points $x$ and $y$ there are $\frac{\left(\begin{array}{l}6 \\ 1\end{array}\right)}{\left(\begin{array}{c}2 \\ 1\end{array}\right)}=3$ tetrads containing both $x$ and $y$, and $7-3=4$ contain $x$ but not $y$. But there are $7-4=3$ tetrads do not contain neither points. For a given triple there is one 201 tetrad contains all three points, and $3-1=2$ tetrads having two points only. The number of tetrads containing one point of the triple $=4-2=2$, but $3-2=1$ 
tetrad with no point from the triple. Now we draw a horizontal line as up to now the calculations are true for any 1,2, and 3 points. However, below the line it is true for "special tetrads" only; four fixed points in the Steiner system. The last line in Table 1 shows that there is only one special tetrad containing four fixed points, and $1-1=0$ tetrads with three points but not the fourth one. Also, we have $2-0=2$ tetrads with two of the four points, and $2-2=0$ tetrads with one point only. The number of tetrads that do not contain any of the four points is $1-0=1$. By continuing in this way we get Table 1;

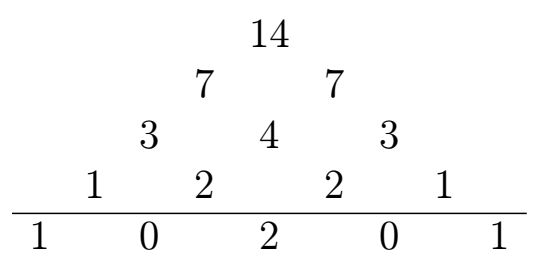

Table 1: The intersection triangle of the Steiner system $S(3,4,8)$

We conclude from the last line of the diagram that any two tetrads are either disjoint or intersect in two points, and also the complement of a tetrad is a tetrad.

Let $\{\infty 013\},\{\infty 045\},\{\infty 062\},\{\infty 124\},\{\infty 235\},\{\infty 346\},\{\infty 561\}$ be all the tetrads containing $\infty$. Then from Table 1 the complement of these tetrads are also tetrads, i.e. $\{1345\},\{4562\},\{1362\},\{0365\},\{1406\}$, $\{2510\},\{0234\}$ are also special tetrads. We confirm that the permutation $\alpha=(\infty 0)$ (1 3) (4 5) (6 2) preserves $\mathcal{S}$. It is of interest that the intersection triangle for an $S(3,4,8)$ implies the following Lemma, although we shall not need to use the Lemma in our construction.

Lemma 2.2 if $T, U \in \mathcal{S}$ and $|T \cap U|=2$, then $T+U \in \mathcal{S}$

Proof. By $T+U$ we mean the symmetric difference which is $(T \cup U) \backslash(T \cap U)$ $=(T \backslash U) \cup(U \backslash T)$.

So, let

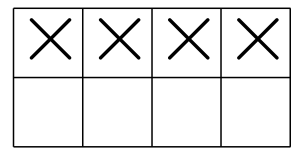

and

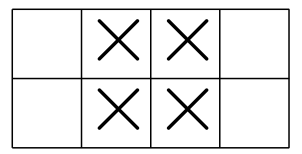

are tetrads

$X=\left\{a_{1}, a_{2}, a_{3}, a_{4}\right\} \in \mathcal{S}$ and Let $Y=\left\{a_{1}, a_{2}, b_{1}, b_{2}\right\} \in \mathcal{S}$. We want to find the tetrad 312 containing $\left\{a_{3}, a_{4}, b_{1}\right\}$. As the last line of Table 1 implies that two tetrads are either intersect in two points or they are disjoint, it follows that the new tetrad cannot have $a_{1}$ nor $a_{2}$. Hence, the new tetrad has to intersect 


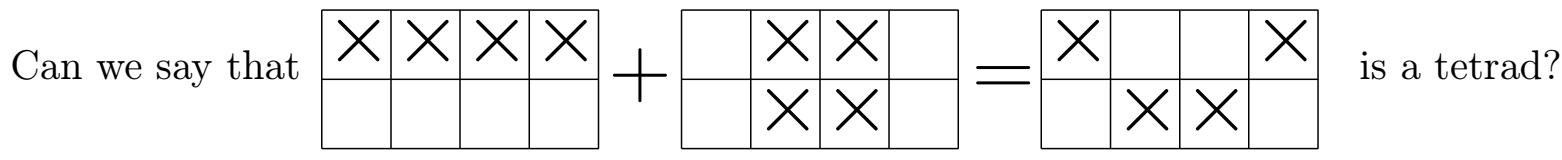

$\begin{array}{llllll}\infty 124 & \longrightarrow & \longrightarrow & 0365 \\ \infty & 235 & \longrightarrow & \longrightarrow & 1406 \\ \infty 346 & \longrightarrow & \longrightarrow & 2510 \\ \infty 450 & \circlearrowleft & \circlearrowright & 3621 \\ \infty 561 & \longrightarrow & \longrightarrow & 4032 \\ \infty 602 & \circlearrowleft & \circlearrowright & 5143 \\ \infty 013 & \circlearrowleft & \circlearrowright & 6254\end{array}$

Table 2: Tetrads of Steiner system $S(3,4,8)$

with $Y$ in two points. Therefore, the new tetrad is $\left\{a_{3}, a_{4}, b_{1}, b_{2}\right\}$, which is the symmetric difference of $X$ and $Y$.

Remark: Let $\{123 x\}$ be a special tetrad what is the value of $x$ ?

Proof. As this is a special tetrad, it cannot intersect with any other tetrad in more than two points. Hence, $x \notin\{\infty, 4,5,0\}$. It follows that $x=6$.

Proposition 2.3 If there is a Steiner system $S(3,4,8)$ then every triple exists in one tetrad only, and $\operatorname{Stab}(S(3,4,8)) \cong 2^{3}: L_{3}(2)$.

Proof. We shall take $\infty$ along with $\mathbb{Z}_{7}$, then fixing $\infty$ we get an $S(2,3,7)$, i.e take all the tetrads containing $\infty$ in Table 2 and consider the triples in these tetrads, we have $\{124,235,346,450,561,602,013\}$, which is a Steiner system $S(2,3,7)$. Note that fixing any point will give us $S(2,3,7)$. From the intersection triangle of $S(3,4,8)$ the complement of a tetrad is a tetrad so we get the compete table. Now we have a list of 14 tetrads with the property that any triple exists in one tetrad only. Hence, we have constructed $S(3,4,8)$. Next to find the group that preserves this system let us produce a permutation that maps tetrads in Table 2 among themselves. 349 Let $\rho=(\infty 0)(13)(45)(26)$ and apply $\rho$ on $\mathcal{S}=\{\infty 124, \infty 235, \infty 346, \infty 450, \infty 561, \infty 602, \infty 013\}$, we get Table 2: 
Hence $\rho$ preserves this system. So, $\rho \in \widehat{L}$, where, $\widehat{L}=\left\{\sigma \in S_{8}: \mathcal{S}^{\sigma}=\mathcal{S}\right\}$. Then $376|\widehat{L}|=8 \times L_{\infty}=8 \times 168$. As the group

$$
<(\infty 0)(13)(45)(26),(\infty 1)(24)(56)(30),(\infty 3)(01)(25)(64)>
$$

elementary abelian of order $2^{3}$, it follows that $\widehat{L} \cong 2^{3}: L_{3}(2)$.

\subsection{Steiner System $\mathrm{S}(5,6,12)$ and The Kitten}

In this section we introduce the following observations:

We will start with the construction of Steiner system $S(5,6,12)$. Then we will prove that this system exists depending on the proof by Havlicek and Lenz in [8]. Let $\mathcal{P}$ be the set of points of the projective plane of order 3, i.e. Steiner system $S(2,4,13)$. From the properties of a projective plane this plane contains 13 points and lines, where each line contains 4 points and every pair of distinct points lies in a unique line. Now, we will introduce four types of sets $\mathfrak{L} \subset \mathcal{P}$, where each type consists of 6 points:

1. $\mathfrak{L}$ is the union of a line and two further points not on the line. This set contains a unique line so there are exactly $13 \times\left(\begin{array}{c}13-4 \\ 2\end{array}\right)=468$ of this type.

2. $\mathfrak{L}$ is the symmetric difference of two different lines, i.e. the union of two lines less their intersection. We can write this difference in one way only; $\frac{13 \times 12}{2 !}$. Hence there are 78 of this type.

3. $\mathfrak{L}$ consists of a triangle and an inscribed triangle, i.e. each point of the second triangle lies on exactly one line of the first triangle. In this case each vertex of the big triangle is on exactly two trisecants of $\mathfrak{L}$, whereas each point of the inscribed triangle is on one trisecant only. Since two distinct vertices of the inscribed triangle determine the third one uniquely, it follows that we have $\frac{13 \times 12 \times(13-4)}{3 !} \times 2 \times 2=936$ of this type.

4. Consider the set of vertices of a quadrilateral; the set of 4 points where no 3 are collinear. In this case $\mathfrak{L}$ is the set of points where two distinct lines of the quadrilateral meet. So, we count the number of four trisecants $=\frac{13 \times 12 \times(13-4)(13-9)}{4 !}=234$ of this type.

As these four types of 6 -sets are all distinct, it follows that we have $468+$ $78+936+234=17166$-sets. Note that $\left(\begin{array}{c}13 \\ 6\end{array}\right)=1716$. Hence, our system contains all possible 6-sets.

Now let $\alpha$ be a point in $\mathcal{P}$ and let $\mathcal{W}=\mathcal{P} \backslash\{\alpha\}$. So, $\mathcal{W}$ has 12 points and we can Define a block $B$ such that $B \subset \mathcal{W}$ satisfies one of the following:

1. $B$ is the symmetric difference of two distinct lines neither containing $\alpha$. The number of blocks of this type is $\frac{(13-4) \times(13-5)}{2 !}=36$. 
2. $B \cup \alpha$ is the union of two distinct lines. Now we have to consider two cases;

(a) $\alpha$ is on both lines and so we have $\left(\begin{array}{l}4 \\ 2\end{array}\right)=6$

(b) $\alpha$ is not on both lines and so we have $4 \times(13-4)=36$

Hence, in total there are 42 blocks.

3. $B$ consists of a quadrangle with two of its diagonal points and $\alpha$ is the remaining point. If we draw two distinct lines $A$ and $C$ through $\alpha$, then choose two distinct points on $A \backslash \alpha$ and $C \backslash \alpha$ respectively. Therefore the number of blocks $=\left(\begin{array}{l}4 \\ 2\end{array}\right) \times\left(\begin{array}{l}3 \\ 2\end{array}\right) \times\left(\begin{array}{l}3 \\ 2\end{array}\right)=54$.

To sum up we have a total of $36+42+54=132$ blocks. Now we can state our main result;

Theorem 2.4 The set $\mathcal{W}$, together with the set of all blocks, is a Steiner system $S(5,6,12)$.

Proof. By construction all blocks have size 6. We will show that each 5-set $\mathcal{M}$ in $\mathcal{W}$, where we have $\left(\begin{array}{c}12 \\ 5\end{array}\right)=792$ of them, belongs to at least one block. Consider $\mathcal{N}=\mathcal{M} \cup \alpha$ as a 6 -point set, N.B $\alpha$ is not in $\mathcal{M}$, then there are four types of $\mathcal{N}$;

1. Suppose that $\mathcal{N}$ consists of a line $A$ and two other points. Let $B$ be the line joining these two points. Then $(A \cup B) \backslash \alpha$ is a block of type (2) containing $\mathcal{M}$.

2. Let $\mathcal{N}$ be the symmetric difference of distinct lines $A$ and $C$. Recall that 4 points in $P G_{2}(3)$ lie on each line and two lines can only intersect in one point [9]. Now, $(A \cup C) \backslash\{\alpha\}$ is a block of type (2).

3. Assume that $\mathcal{N}$ is a union of a triangle $\{A, B, C\}$ and an inscribed triangle $\{P, Q, R\}$ such that $P \in B C, Q \in C A$ and $R \in A B$. Now we have two cases for $\alpha$ whether it satisfies one of the following; if $\alpha$ lies on $\{P, Q, R\}$, let $R=\alpha$, and consider $X=A P \cap B Q$. Then $X \in C R$ and the set of points $\{A, B, C, X\}$ is a quadrangle with diagonal points $P, Q$. This is exactly a block of type (3) containing $\mathcal{M}$ Figure $1(1)$. Otherwise, $\alpha \in\{A, B, C\}$. Let $C=\alpha$ and consider $X=P Q \cap R C$. Then it follows that $X \in A B \cup P Q$. So, the symmetric difference of $A B$ and $P Q$ is a block of type (1) containing $\mathcal{M}$, Figure 1(2).

4. Let $\mathcal{N}=\{A, B, C, D, E, F\}$ be the set of vertices of a quadrangle. WLOG, let $\alpha=F=A B \cap C D$. This means that $\{A, B, C, D\}$ is a quadrangle with diagonal points $E, \alpha$ and some point $X$. So, $\{A, B, C, D, E, X\}$ is a block of type (3) containing $\mathcal{M}$, Figure $1(3)$. 
Now consider the number of blocks containing our 5-set $\mathcal{M}$. Each of the 132 blocks contain exactly 6 subsets of $\mathcal{W}$ with 5 elements. Thus if we sum over our 5 -sets $\mathcal{M}$ we get $132 \times 6=792$. However we showed that any 5 -set $\mathcal{M}$ is contained in at least one block, it follows that the number of blocks is greater than or equal to 1 for each 792 possible sets $\mathcal{M}$. Thus the number of blocks is equal to 1 for all $\mathcal{M}$.

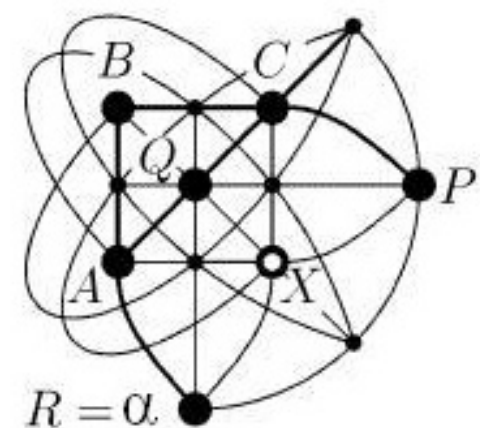

(1)

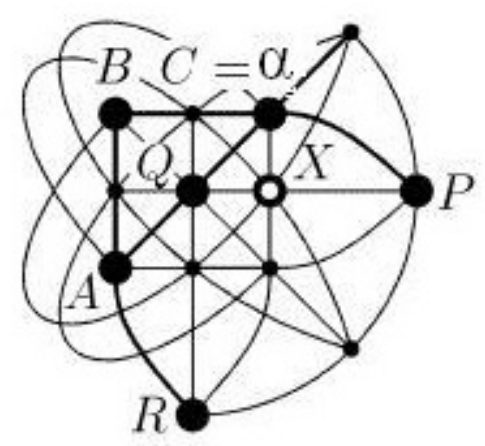

(2)

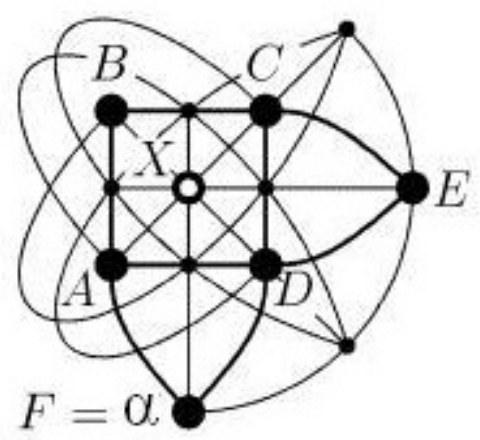

(3)

Figure 1: Steiner system $S(5,6,12)$.

Remark: Conway and Curtis [5] developed an elegant way to construct hexads in the Steiner system $S(5,6,12)$ by gluing together three affine planes, which are simply Steiner sysytems $S(2,3,9)$. The 12 points are labelled using the projective line. Let

$$
P G(1,11)=\{\infty, 0,1,2,3,4,5,6,7,8,9, X\}
$$

we write $X$ for 10 . Take the set of quadratic residues mod 11 together with $0, Q=\{0,1,3,9,5,4\}$. Let $L=<\alpha, \beta>\cong P S L(2,11)$, where $\alpha$ and $\beta$ are permutations such that $\alpha(y)=y+1$ and $\beta(y)=\frac{-1}{y}$. Now, $\mathcal{L}=\left\{Q^{x} \mid x \in L\right\}$ consists of 132 subsets of size 6 , the so called hexads. These hexads have the property that any five of the 12 points occur together in just one of them. Hence, $\mathcal{L}$ form the Steiner system $(5,6,12)$.

Curtis [5] calls his method the Kitten. The construction of the Kitten is based on the fact that given three points, $\{\infty, 0,1\}$ say, then the triples which completes this to a hexad form a Steiner system $S(2,3,9)$, i.e. an affine plane. There is only one such plane Figure 2, where the lines of this system are shown as rows, columns and generalized diagonals of a "noughts-and-crosses" board.

The number of lines $=\frac{\left(\begin{array}{l}9 \\ 2\end{array}\right)}{\left(\begin{array}{c}3 \\ 2\end{array}\right)}=12$ lines. From Figure 2 these lines partition into 4 sets of parallel lines also the rows and columns are perpendicular to each 


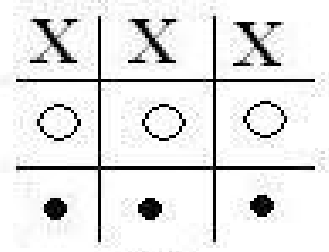

(0)

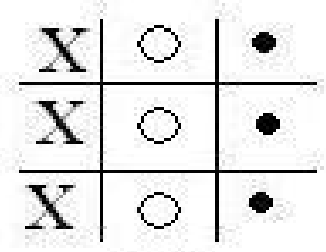

$(\infty)$

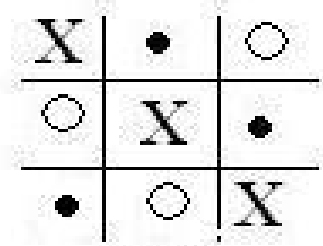

(-1)

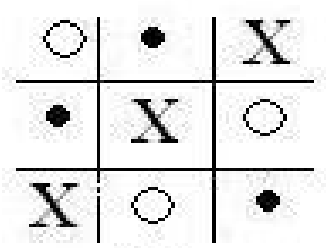

(1)

Figure 2: Slope of the lines of Steiner system $S(2,3,9)$.

other as are the 2 families of diagonal lines. The union of two perpendicular lines is a cross where we have $\frac{12 \times 3}{2}$ crosses, and the complement of a cross is a square, see Figure 3 where the $X$ s form crosses and dots form squares.
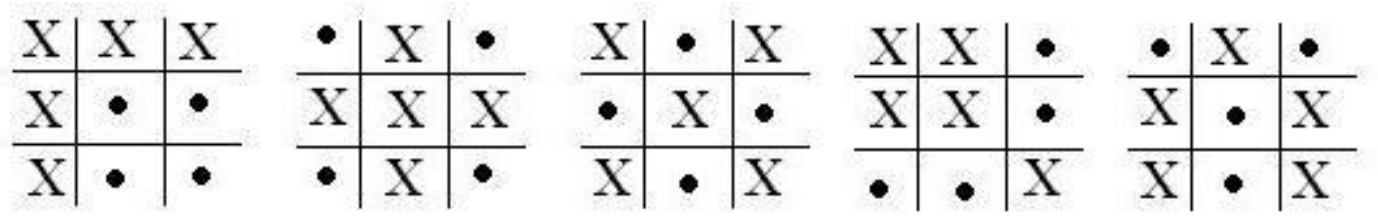

Figure 3: Crosses and Squares of the Steiner system $S(2,3,9)$

To construct the Kitten we start by drawing a triangle with vertices labeled $\infty, 0,1$. Then write $6,7,8$ down the $\infty$-altitude. The next step is to perform anticlockwise rotation through $120^{\circ}$ correspond to the linear fractional permutation on both large and small triangles.

$$
R: y \longmapsto \frac{1}{1-y} \equiv(\infty 01)(2 X 6)(358)(479)
$$

Note that this permutation is performed modulo 11 and we are writing $X$ for 10 .

$$
\begin{array}{c|c|c}
6 & X & 3 \\
\hline 2 & 7 & 4 \\
\hline 5 & 9 & 8
\end{array}
$$

\begin{tabular}{c|c|c}
5 & 7 & 3 \\
\hline 6 & 9 & 4 \\
\hline 2 & $X$ & 8
\end{tabular}

$$
\begin{array}{c|c|c}
5 & 7 & 3 \\
\hline 9 & 4 & 6 \\
\hline 8 & 2 & X
\end{array}
$$

Table 3: $\infty, 0,1$ pictures

We call $\{\infty, 0,1\}$ the line at $\infty$, and $\infty, 0$ and 1 are points at $\infty$. Now we can easily see the hexads and they are: 
- $\{\infty, 0,1\} \cup$ any line.

- The union of two parallel lines.

- A point at $\infty$ together with a cross in the corresponding picture.

- Two points at $\infty$ and a square in the picture corresponding to the omitted point at $\infty$.

Example 2.5 Find the hexads containing $\{3,5,7,9,0\}$, and $\{2,3,4,5,6\}$

To find the first hexad we look at the picture at 0 and find that $X$ gives us a cross, hence the hexad is $\{3,5,7,9,0, X\}$. Looking at the picture at 0 to find the next hexad we find that 8 completes the line to get two parallel lines. So, $\{2,3,4,5,6,8\}$ is a hexad.

Since this Kitten has all the hexads in $S(5,6,12)$ which are preserved by $M_{12}$, it follows that the three reflections in the altitudes, $f_{\infty}, f_{0}$, and $f_{1}$ must be members of $M_{12}$.

If we let $\alpha=(123456789 X)$ then:

- $<\alpha, f_{\infty}>\cong M_{11}$ fixing $\infty$

- $<\alpha, f_{0}>\cong M_{11}$ transitive on the 12 letters

- $<\alpha, f_{1}>\cong M_{12}$

\subsection{The Steiner System $S(5,8,24)$, the MOG, and the Hexacode}

A Steiner system $S(5,8,24)$ is a collection of 8-element subsets, called octads, of a 24 -element set, $\Omega$, where each five elements of $\Omega$ lie in just one octad. If such a system exists then the number of octads $=\frac{\left(\begin{array}{c}24 \\ 5\end{array}\right)}{\left(\begin{array}{l}8 \\ 5\end{array}\right)}=759$ octads. This time we will use the Golay code to prove existence of the Steiner system $S(5,8,24)$.

Definition 2.6 [7] Let $F$ denote a finite field and $n \geqslant 1$. A linear code $\mathcal{C}$ is a vector subspace of the vector space $V=F^{n}$ for some $n \geqslant 1$. The elements of $\mathcal{C}$ are called codewords and $n$ is the length of $\mathcal{C}$.

The dimension of the code is the dimension of the subspace that forms the linear code. We will consider codes over the following fields:

- $\mathbb{F}_{2}=\{0,1\}$ the binary field;

- $\mathbb{F}_{3}=\{0,1,-1\}$ the ternary field;

- $\mathbb{F}_{4}=\{0,1, \omega, \varpi\}$ the field of order four. 
Let $\Omega=\left\{\omega_{1}, \omega_{2}, \ldots, \omega_{n}\right\}$ and let $V=\mathcal{P}(\Omega)$, the powerset of $\Omega$. Consider an element $A$ in $V$ we Define the weight of $A$ is the cardinality of $A$. Suppose that $A$ and $B$ are two elements in $V$, then the addition of vector spaces is replaced by the symmetric difference of the subsets, i.e. $(A \cup B)-(A \cap B)$.

Definition 2.7 (Chapman) [2] A binary Golay code, is a binary linear code of length 24 , of dimension at least 12 , and a minimum weight at least 8.

Theorem 2.8 [2] Let $\Omega=\left\{\omega_{1}, \omega_{2}, \ldots, \omega_{24}\right\}$ and let $V=\mathcal{P}(\Omega)$, the powerset of $\Omega$. If $\mathcal{C}$ is a binary Golay code then:

1. $\mathcal{C}$ has dimension 12 ,

2. $\mathcal{C}$ has minimum weight 8 ,

3. the words of weight 8 in $\mathcal{C}$ form an $S(5,8,24)$ Steiner system,

To keep the paper self contained we present the:

Proof. We consider congruences in $V$ modulo $\mathcal{C}$. Fix an element $\omega \in \Omega$. Let $S_{\omega}$ be the set of $A \in V$ with $|A| \leqslant 4$ and with $\omega \in A$ if $|A|=4$. Then $\left|S_{\omega}\right|=\left(\begin{array}{c}24 \\ 0\end{array}\right)+\left(\begin{array}{c}24 \\ 1\end{array}\right)+\left(\begin{array}{c}24 \\ 2\end{array}\right)+\left(\begin{array}{c}24 \\ 3\end{array}\right)+\left(\begin{array}{c}23 \\ 3\end{array}\right)=1+24+276+2024+1771=4096=2^{12}$. The sum of two elements of $S_{\omega}$ has weight at most $4+4=8$. However, if this is true then each element should have weight 4 so, $\omega$ would be in both of them, this implies that their sum, symmetric difference, is at most 6 not 8 . Therefore, the sum of two distinct elements of $S_{\omega}$ cannot lie in $\mathcal{C}$. The cosets $A+\mathcal{C}$ as $A$ runs through $S_{\omega}$ are all distinct. Thus $|V / \mathcal{C}| \geqslant 2^{12}$. But as $|\mathcal{C}| \geqslant 2^{12}$ then $|V / \mathcal{C}| \leqslant \frac{2^{24}}{2^{12}}=2^{12}$ and so $|\mathcal{C}|=|V / \mathcal{C}|=2^{12}$. Thus $\mathcal{C}$ has dimension 12. Also the $A+\mathcal{C}$ for $A \in S_{\omega}$ form a complete set of cosets of $\mathcal{C}$ in $V$.

Let $B \in V$ have weight 4 , and suppose that $\omega \notin B$. Then $B \notin S_{\omega}$, but $B \in A+\mathcal{C}$ for some $A \in S_{\omega}$. As $A+B$ is a non-zero element of $S_{\omega}$, then $A+B$ has weight 8 , and so $\mathcal{C}$ has minimum weight 8 . The set $A+B$ contains $\{\omega\} \cup B$. Thus each 5-element subset of $\Omega$ containing $\omega$ is contained in at least one 8element set in $\mathcal{C}$. But as $\omega$ is an arbitrary element of $\Omega$ then each 5-element subset of $\Omega$ is contained in at least one 8 -element set of $\mathcal{C}$. But this element is unique; any pair of distinct 8-element sets $A, B \in V$ with $|A \cap B| \geqslant 5$ elements satisfy $0<|A+B| \leqslant 6$, contrary to $\mathcal{C}$ s having minimum weight 8 . Note that $|A+B|=|A|+|B|-2|A \cap B|$. Thus the weight 8 words of $\mathcal{C}$ form a Steiner system $\mathrm{S}(5,8,24)$ and so there are $\frac{\left(\begin{array}{c}24 \\ 5\end{array}\right)}{\left(\begin{array}{l}8 \\ 5\end{array}\right)}=\frac{42504}{56}=759$ words of weight 8 in $\mathcal{C}$.

Remark: This shows that a binary Golay code determines a Steiner system $S(5,8,24)$. 
Now consider a Steiner system $S(3,4,16)$. There are $\frac{\left(\begin{array}{c}16 \\ 3\end{array}\right)}{\left(\begin{array}{c}4 \\ 3\end{array}\right)}=\frac{16 \times 15 \times 14}{4 \times 3 \times 2}=140$ tetrads in this system. Let $V$ be a vector space of dimension 4 over $G F_{2}$ and consider the two dimensional subspaces of $V$. There are $\frac{\left(2^{4}-1\right)\left(2^{4}-2\right)}{\left(2^{2}-1\right)\left(2^{2}-2\right)}=$ 35 two dimensional subspaces of $V$. Let $U \leqslant V$ with $\operatorname{dim} U=2$. Then $V=U+\left(U+v_{1}\right)+\left(U+v_{2}\right)+\left(U+v_{3}\right)$ for some $v_{1}, v_{2}, v_{3} \in V$. Let $\mathcal{S}=$ $\{U+v \mid U \leqslant V, \operatorname{dim} U=2, v \in V\}=\{\{u, v, w, u+v+w\} \mid u, v, w$ distinct $\}$ be the set of tetrads then $|\mathcal{S}|=35 \times 4=140$.

Curtis [4] constructed the Miracle Octad Generator (MOG) to read off the octads in the Steiner system $S(5,8,24)$. The MOG is a one-to-one correspondence between 35 partitions of the Steiner system $S(3,4,16)$ and the partitions of an 8-set into 4-sets $\left(\begin{array}{l}8 \\ 2\end{array}\right) \times \frac{1}{2}=35$.

The MOG consists of three $4 \times 2$ bricks, which are themselves octads of the $S(5,8,24)$. Such a union of 3 disjoint octads is called a trio and since, octads intersect evenly; an octad must cut a cross a trio as 800,440 or 422 . In the latter case, which is the most common, we call the octad containing 4 points the heavy brick. The other two are called the square. Since the union of any 2 columns of the square form an octad, and the union of the rows of the square form an octad, any other octad must intersect every row with the same parity and every column with the same parity, see Example 2.10.

The brick tetrad can be any one of the $\left(\begin{array}{l}8 \\ 4\end{array}\right)=70$ tetrads which fall into two groups of 35. The square tetrad must be one of the 140 special tetrads. These fall into 35 groups of 4 .

The MOG has 35 pictures which show against each complementary pair of brick tetrads, the corresponding group of special square tetrads. To obtain an octad we take a brick tetrad with any square tetrad of the same picture.

Example 2.9 Find the octad containing the points 0,4,6,11,17 as labelled in Figure 4

Now the heavy brick is the one containing 4,11,17 and in order to find the forth point, check the parity of the bricks. If we choose 2 to be the missing point. Then we notice that in column 5 row 5 of Figure 4, 6 and 0 do not lie in the same brick tetrad. Hence our point is 16 . In column 3 row 2 of the MOG 6 and 0 lie on the same brick tetrad so the octad is $0,4,6,11,16,17,20,22$.

Example 2.10 Find the octad containing the points 1,3, 4, 8, 19 as labelled in Figure 4

We must complete

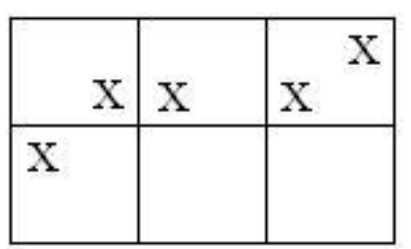

to an octad. First we

need to identify the heavy brick. Suppose that it contains 3 and 8 then the 
corresponding square tetrad is

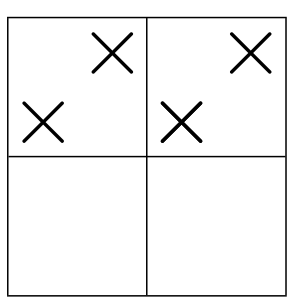

But 3 and 8 do not lie in the same brick; column 2 row 4 of Figure 4 . So, our square must be;

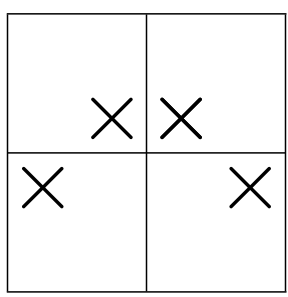

In the third column sixth row of Figure 4 we find out octad to be 1, 3, 4, 6, 7, 8, 9, 19

After Curtis' construction of the MOG, Conway [3] introduced the Hexacode;

Definition 2.11 The Hexacode $\mathcal{H}$ is a linear code of length 6 and dimension 3 over $\mathbb{F}_{4}$.

Note that $\mathbb{F}_{4}$ is the field of four elements $\{0,1, \omega, \varpi\}$ such that $\omega+\varpi+1=0$. Now we may Define $\mathcal{H}$ to be

$$
\mathcal{H}=\left\{(a, b, c, a+b+c, \varpi a+\omega b+c, \omega a+\varpi b+c): a, b, c \in \mathbb{F}_{4}\right\}
$$

Lemma 2.12 There are $2^{6}$ such hexacodes.

Proof. As

$$
\mathcal{H}=<(1,1,1,1,0,0),(0,0,1,1,1,1),(\varpi, \omega, \varpi, \omega, \varpi, \omega)>\text {, then }
$$

$$
\mathcal{H}=\left\{\begin{array}{cc}
(0,0,0,0,0,0), & 1 \text { such } \\
(0,0,1,1,1,1), & 3 \text { (places for } 0) \times 3(\text { choices for powers of } \omega)=9 \text { such } \\
(\varpi, \omega, \varpi, \omega, \varpi, \omega), & 4(\text { column permutations }) \times \\
& 3(\text { choices for the missing element })=12 \text { such } \\
(\varpi, \omega, 0,1,0,1), & 3(\text { place of } \omega, \varpi) \times 3 \text { (powers of } \omega) \times \\
& 2(\text { column permutations for } \omega, \varpi) \\
& \times 2(\text { column permutations for } 0,1)=36 \text { such } \\
(1,1, \omega, \omega, \varpi, \varpi) & 3(\text { powers of } \omega) \times 2(\text { column permutations })=6 \text { such }
\end{array}\right\}
$$




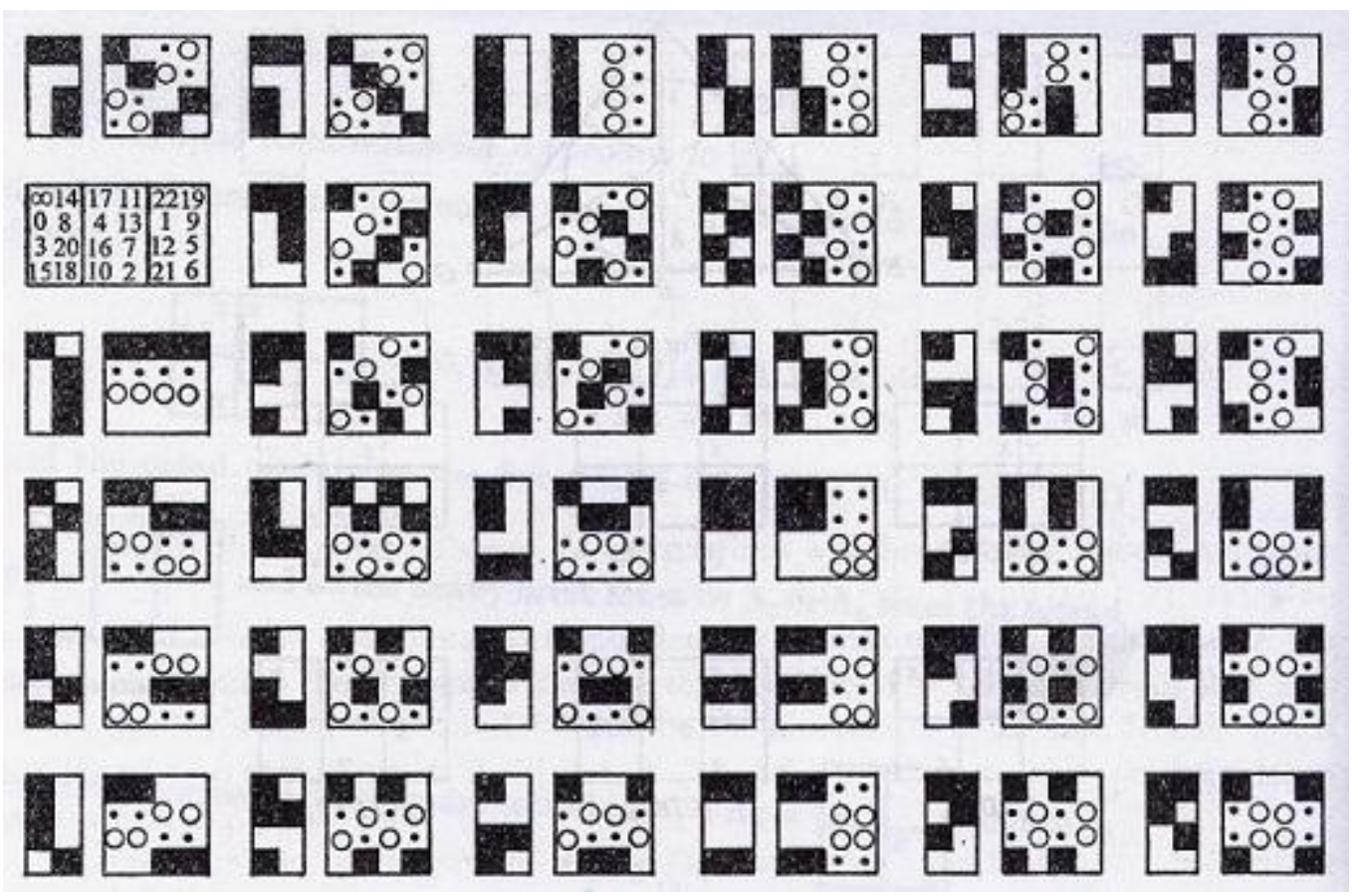

Figure 4: The MOG

Note that multiplication by powers of $\omega$ are allowed, also, $S_{4} \cong 2^{2}: S_{3}$ permutations of the column corresponding to $S_{4} \cong<\left(\begin{array}{lll}1 & 3 & 5\end{array}\right)\left(\begin{array}{lll}2 & 4\end{array} 6\right),(12)(34),(13)(24)>$ preserves the code. Hence, in total we have $1+9+12+36+6=64=2^{6}$ hexacodes.

Observation: If the rows of the MOG are labeled with the elements of $\mathbb{F}_{4} ; 0,1, \omega, \varpi$ respectively. Then there are two different interpretations for the Hexacode $h=\left(h_{1}, h_{2}, \ldots, h_{6}\right)$, where $h_{i} \in 923 \mathbb{F}_{4}, \forall 1 \leqslant i \leqslant 6$; odd and even interpretation. In the odd interpretation Figure5, if $h_{i}=\lambda \in \mathbb{F}_{4}$ we place 1 in the $\lambda$ position in the $i$ th column and zeros in the other three positions. We might take the complement of this, i.e. put zero in the $\lambda$ position and $1 \mathrm{~s}$ in the other positions. We complete this procedure for all $h_{i}$ s considering that we have the right to complement as long as we keep an odd number in the first row.

On the other hand, in the even interpretation Figure 6 , if $h_{i}=\lambda \neq 0$, we place 1 in the 0 th and $\lambda$ th position and zero in the other two positions, or we may take the complement. Otherwise, if $h_{i}=0$ we either place 0 or 1 in four positions. We always have to keep the top row even in this interpretation. 


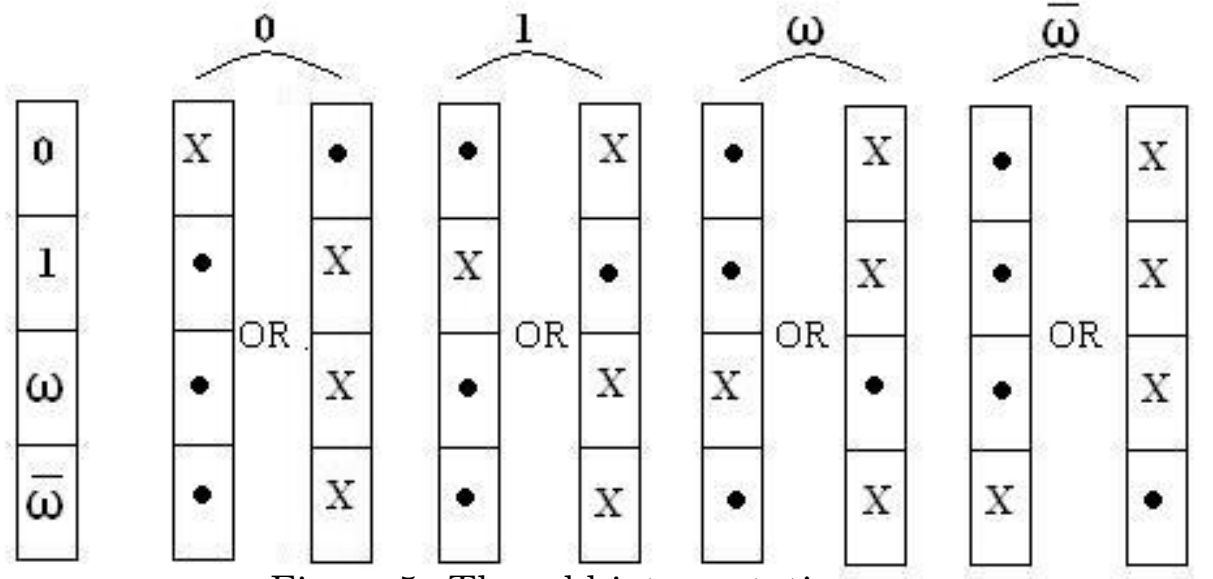

Figure 5: The odd interpretation

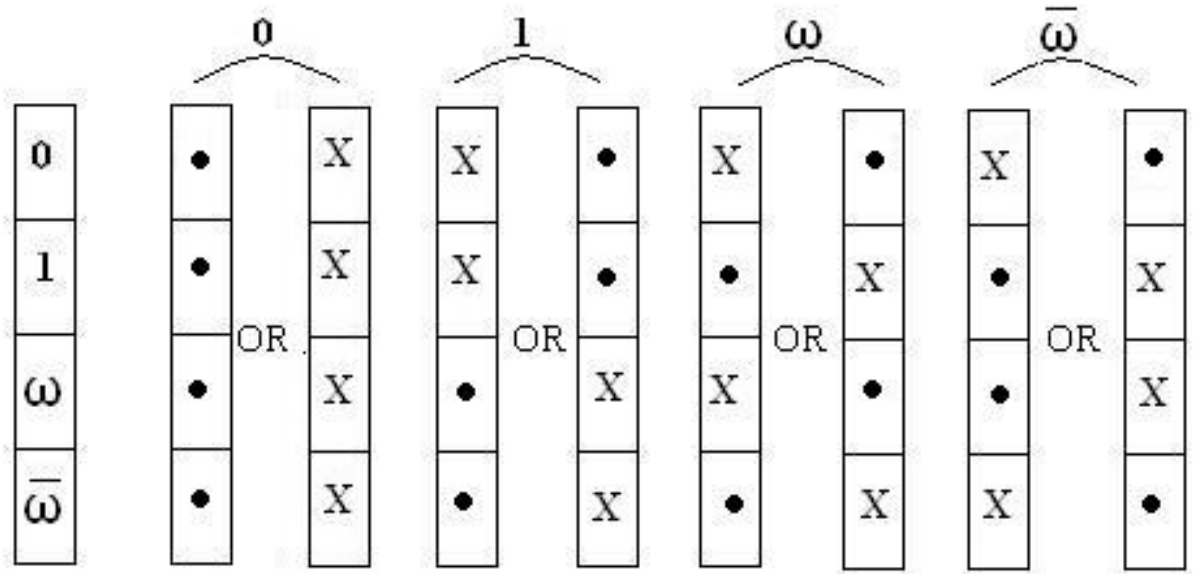

Figure 6: The even interpretation

Note that there are in fact $2^{5}$ odd and $2^{5}$ even interpretation of any hexacode word. So, we have

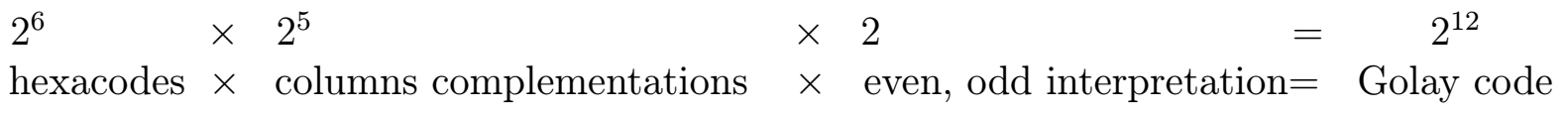

Example $2.13(0,1, \varpi, \omega, 0,1) \sim$ for instance to; 


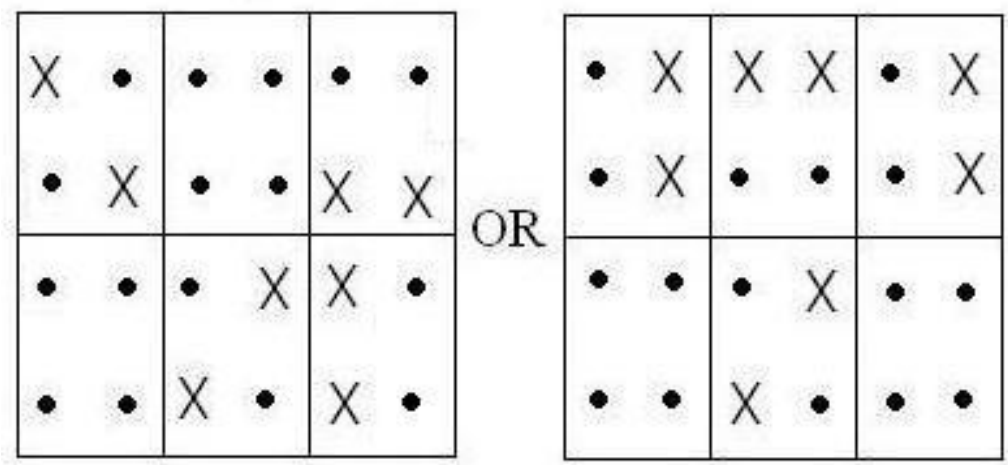

\section{Conclusion}

In this paper we prove that a Steiner system $S(2,3,7)$ can be extended to $S(3,4,8)$. In this Steiner system any two tetrads are either disjoint or intersect in two points. Also, the every triple exists in one tetrad only. The second Steiner system we construct is $S(5,6,12)$. The last one is $S(5,8,24)$.

Acknowledgements. I would like to thank Prof. Robert T. Curtis for his guidance and support.

\section{References}

[1] M. Aschbacher. Sporadic Groups. Cambridge University Press, New York, 1994.

[2] R. Chapman. Constructions of the golay codes: A survey, 1997.

[3] J.H. Conway. Sphere Packing, Lattices and Groups. Springer-Verlag, 1988.

[4] R. T. Curtis. A new combinatorial approch to $m_{24}$. Math. Proc. Cambridge Philos. Soc., 79:25-42, 1976.

[5] R.T Curtis. The steiner system $s(5,6,12)$, the mathieu group $m_{12}$ and the kitten. Computational Group Theory, 1984.

[6] H. Cuypres. The mathieu groups and designes, 1994.

[7] G. Everest. Coding theory, 2003-2004.

[8] H. Havlicek and H. Lenz. Another simple proof for the exeistance of the small witt design. Elem. Math, 56, 2001.

[9] D. R. Hughes and F. C. Piper. Projective Planes. Springer-Verlag, New York, 1973. 
[10] J.J. O'Connor and E.F. Robertson. Jakob steiner. The Mac Tutor History of Mathematics Archive, 1996.

Received: January 30, 2014 